SCIENTIFIC LETTER

\title{
Herpes simplex virus type 1 infection in rheumatic valvar disease
}

\author{
Y Li, Z Pan, Y Ji, H Zhang, L C Archard
}

Heart 2005;91:87-88. doi: 10.1136/hrt.2003.026377

$\mathrm{S}$ ome features of rheumatic valvar disease resemble the histopathology of herpesvirus infection. While the prevalence of rheumatic fever (RF) has declined in developed countries, 15-20 million cases of RF occur each year in developing countries and RF remains the leading cause of acquired heart disease among children and young adults. A resurgence of RF has been observed in both Europe and the USA. ${ }^{1}$ Chronic, acquired valvar disease is thought to be caused by RF, which parallels the incidence of throat infection with group A streptococci (GAS). ${ }^{2}$ However, many patients with RF have no history of streptococcal pharyngitis, most throat infections with GAS do not result in RF, and RF does not follow repeated streptococcal infection at other sites such as skin. It is generally believed that RF results from an autoimmune response to streptococcal $M$ proteins, crossreactive with human tissue, but serum concentrations of creatine kinase or cardiac troponin type 1 (cTnI) do not increase during active rheumatic carditis indicating that antiheart antibodies do not result in significant myocyte injury. Previous attempts to demonstrate streptococcal antigens in valvar tissue in rheumatic heart disease (RHD) were unsuccessful.

Acute pharyngitis is caused more often by viral infection than by streptococcal infection, with herpes simplex virus type 1 (HSV-1) being responsible for about $4 \%$ of all cases of sore throat. GAS, but not group B streptococci, Staphylococcus aureus, nor Candida albicans, adhere to HSV infected cells in vitro. Pre-incubation of infected cells with anti-HSV serum prevents bacterial adherence, indicating that HSV infection can lead to superinfection specifically with GAS. ${ }^{3}$ The cytopathological characteristics of HSV infection are intranuclear inclusions surrounded by a clear halo and multinucleate giant cells accompanied by inflammation, resembling Anitschkow owl-eye cells and Aschoff giant cells, respectively. These are seen in the pathognomonic Aschoff bodies in valvar tissue from RHD, ${ }^{4}$ suggesting that HSV-1 infection may be involved in RHD.

\section{METHODS}

Immunohistochemical staining for HSV-1 antigen and nested polymerase chain reaction (PCR) amplification of HSV-1 genomic DNA with nucleotide sequencing of products were employed in the present study to detect HSV-1 infection in valvar tissue, collected during surgical valve replacement, from 32 patients with chronic RHD. Diagnosis of RHD was based on a history of RF, echocardiography, electrocardiography, and histopathological changes. Patients were aged from 15-54 years (mean 44 years), 11 were male and 21 female. The duration of illness was more than one year. HSV-1 infected human embryo lung fibroblasts or human lung tissue (DAKO Ltd, Cambridge, UK) served as positive controls. Valvar tissue from five young adults (three male, two female) who died suddenly without valvar disease was used for comparison.
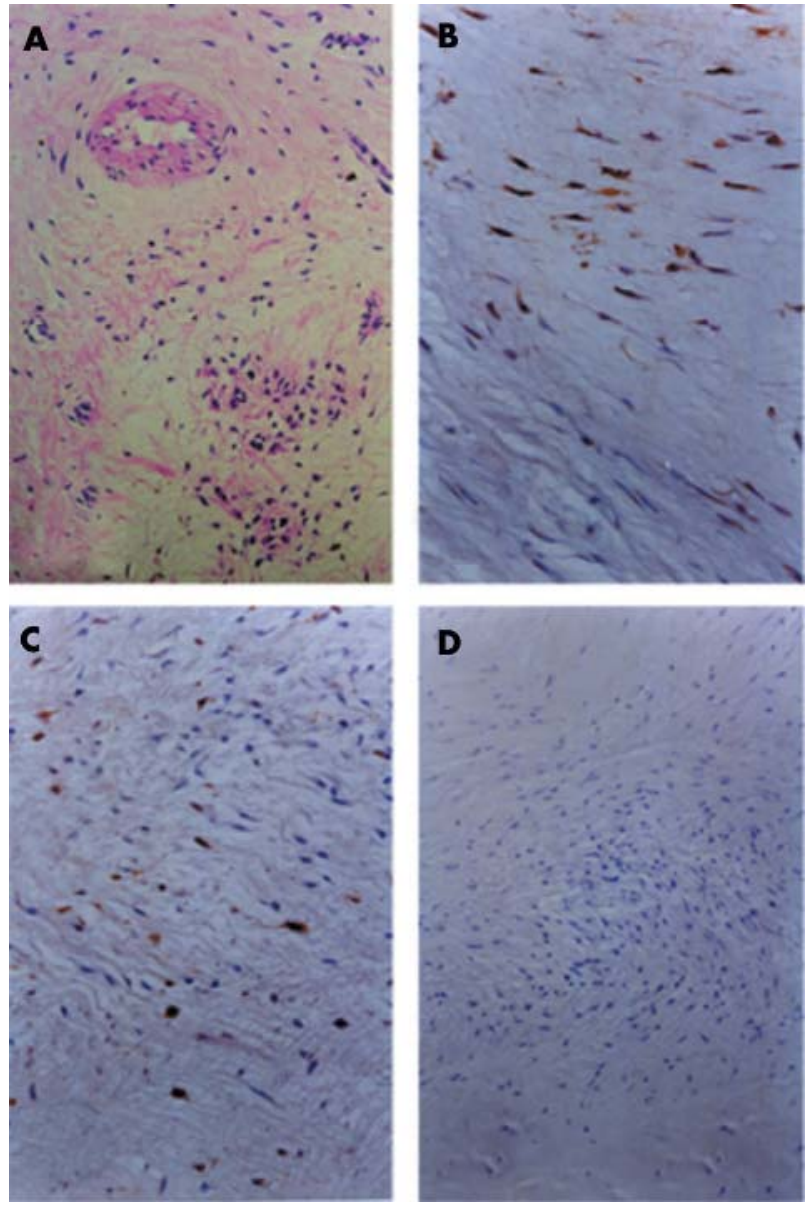

Figure 1 Histopathology and immunohistochemical staining of RHD tissue samples. (A) Haematoxylin and eosin staining showing neovascularisation with scattered inflammatory infiltration $(\times 20)$. (B) Immunostaining of HSV-1 antigens in experimentally infected fibroblasts in culture (brown deposits, $\times 40$ ). (C) Immunostaining of HSV-1 antigen in valvar tissue, signal in fibroblast-like cells, and some possible interstitial cells $(\times 40)$. (D) Immunostaining with universal negative control reagent for mouse, no positive signals in a consecutive section of $C(\times 10)$.

A monoclonal antibody prepared using HSV-1 strain Stoker as antigen was obtained from Vector Lab Ltd (Newcastle, UK); this antibody does not cross react with herpes simplex virus type 2 (HSV-2), varicella zoster virus (VZV), primary isolates of human cytomegalovirus (CMV), adenoviruses,

Abbreviations: CMV, cytomegalovirus; GAS, group A streptococci; HSV-1, herpes simplex virus type 1; HSV-2, herpes simplex virus type 2; $P C R$, polymerase chain reaction; RF, rheumatic fever; RHD, rheumatic heart disease; VZV, varicella zoster virus 


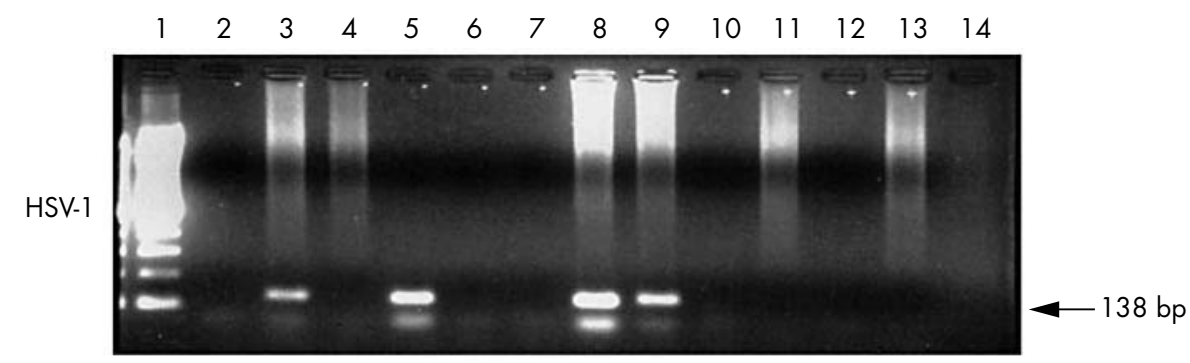

Figure 2 Gel electrophoresis of PCR products. HSV-1 (upper panel) and $\beta$ globin specific (lower panel) amplicons resolved in $2 \%$ agarose gels and stained with ethidium bromide. Lane 1: 100 base pair DNA ladder; lanes 2-11: RHD cases; lanes 12 and 13: sudden death cases; lane 14: reagent control.

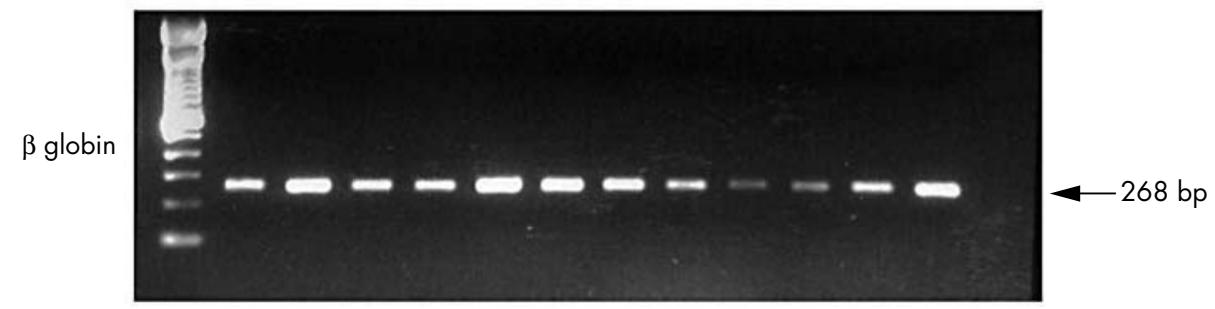

mumps virus, measles virus, or respiratory syncytial virus. An additional, rabbit polyclonal antiserum against HSV-1, Nuniversal negative control reagents for mouse (cocktail of mouse immunoglobulin $G_{1}$, immunoglobulin $G_{2 a}$, immunoglobulin $G_{2 b}$, immunoglobulin $G_{3}$ and immunoglobulin $M$ ) or rabbit (immunoglobulin fraction of serum from non-immunised rabbits), serum-free protein blocking buffer, antibody diluent, and the EnVision detection system were purchased from DAKO Ltd. DNA was extracted from mock infected or HSV-1 infected human embryo lung fibroblasts or from sections of paraffin embedded valvar tissue, using the DNeasy tissue kit (Qiagen Ltd, West Sussex, UK). The PCR primer sets amplify a 138 base pair conserved region of the glycoprotein D gene of HSV-1 which has no significant homology with CMV, Epstein-Barr virus, or VZV DNA, and are suboptimal for amplification of the equivalent HSV-2 sequence. $^{5} \beta$ Globin gene sequences were amplified from each sample as internal controls for DNA preparation, in addition to reagent controls using the forward and reverse primers.

\section{RESULTS}

Fibrosis with vascularisation and scattered lymphocytic and plasma cell infiltration without myxomatous change in valvar tissue sections stained with haematoxylin and eosin was seen in all cases (fig 1A), supporting the clinical diagnosis of RHD. Immunocytochemical staining with the monoclonal antibody specific for HSV-1 antigen was localised in the cytoplasm and nucleus of experimentally infected fibroblasts in culture (fig 1B). Positive signals for virus antigen were seen in tissue from 21 of $32(65.6 \%)$ cases of RHD, mainly in fibroblast-like cells (fig lC), in some possible interstitial cells and occasionally in endothelial cells and smooth muscle cells in neovascularisation. Similarly distributed signals were seen after staining with the rabbit polyclonal antiserum against HSV-1 (not shown). Mock infected or HSV-l infected controls stained with HSV-l antibodies or with $\mathrm{N}$-Universal negative control reagents or with dilution buffer only, gave the anticipated results, confirming the specificity of immunostaining for HSV-I antigen (fig 1D). HSV-1 antigen was also found in valvar tissue from one case in the comparison group.

Two stage nested PCR generated the predicted HSV-1 138 base pair amplicon in 15 of the $32(46.9 \%$ ) cases (fig 2). All 15 cases were also positive for viral antigen. HSV-1 DNA was not found in any tissue sample from the comparison group. The $\beta$ globin gene sequence ( 268 base pair product) was amplified successfully from all samples from both groups. Nucleotide sequence of amplified HSV-1 DNA, determined by direct automated cycle sequencing in both orientations, was homologous with the glycoprotein D gene of HSV-1 (GenBank Accession No. E00401).

\section{DISCUSSION}

This study explores HSV-1 infection in chronic valvar disease and shows the presence of virus antigen and DNA in valvar tissue from a substantial proportion of patients with RHD. Streptococcal involvement may be secondary to HSV infection, with local tissue damage and inflammatory cytokines produced in response to GAS infection being potential factors in reactivation of latent HSV. It is not clear whether HSV-1 infection contributes to valvar damage through autoimmunity or non-immune mechanisms, but this study provides new evidence of a virus role in the pathogenesis of some chronic, acquired valvar disease.

\section{Authors' affiliations}

Y Li, H Zhang, L C Archard, Division of Biomedical Sciences, Faculty of Medicine, Imperial College of Science, Technology \& Medicine, London, UK

Z Pan, Department of General Practice, Zhongshan Hospital, Fudan University, Shanghai, PR China

Y Ji, Department of Pathology, Zhongshan Hospital, Fudan University

Correspondence to: Professor Leonard C Archard, Division of Biomedical Sciences, Faculty of Medicine, Imperial College of Science, Technology \& Medicine, Sir Alexander Fleming Building, Exhibition Road, London SW7 2AZ, UK; I.archard@imperial.ac.uk

Accepted 13 July 2004

\section{REFERENCES}

1 Stollerman GH. Rheumatic fever. Lancet 1997;349:935-42.

2 Cunningham MW. Pathogenesis of group A streptococcal infections. Clin Microbiol Rev 2000;13:470-511.

3 Farber PA, Long WK. Adherence of streptococci to cells infected with herpes virus. Oral Surg Oral Med Oral Pathol 1983;55:463-9.

4 Laurence JF, Hopkins RA. Aschoff bodies in an operatively excised mitral valve. Cardiovasc Pathol 1997;6:231-6.

5 Aurelius $\mathbf{E}$, Johansson B, Skoldenberg B, et al. Rapid diagnosis of herpes simplex encephalitis by nested polymerase chain reaction assay of cerebrospinal fluid. Lancet 1991;337:189-92. 